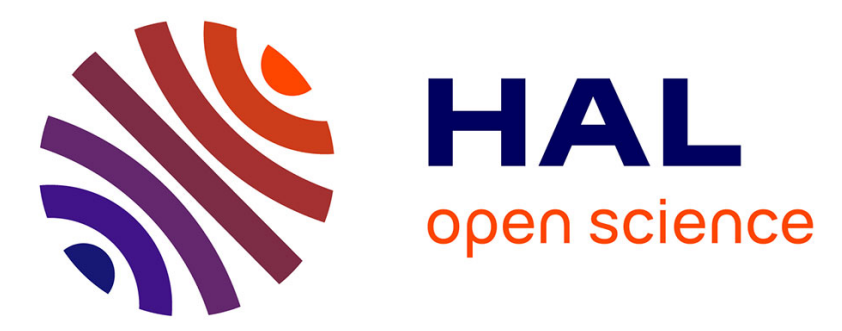

\title{
Decoupled control of the high mobility robot hylos based on a dynamic stability margin
}

Guillaume Besseron, Christophe Grand, Faïz Ben Amar, Philippe Bidaud

\section{To cite this version:}

Guillaume Besseron, Christophe Grand, Faïz Ben Amar, Philippe Bidaud. Decoupled control of the high mobility robot hylos based on a dynamic stability margin. IROS'08: the IEEE/RSJ Int. Conf. on Intelligent Robots and Systems, 2008, Nice, France. pp.2435-2440, 10.1109/IROS.2008.4651092 . hal-03177941

\section{HAL Id: hal-03177941 \\ https://hal.science/hal-03177941}

Submitted on 24 Mar 2021

HAL is a multi-disciplinary open access archive for the deposit and dissemination of scientific research documents, whether they are published or not. The documents may come from teaching and research institutions in France or abroad, or from public or private research centers.
L'archive ouverte pluridisciplinaire HAL, est destinée au dépôt et à la diffusion de documents scientifiques de niveau recherche, publiés ou non, émanant des établissements d'enseignement et de recherche français ou étrangers, des laboratoires publics ou privés. 


\title{
Decoupled control of the high mobility robot Hylos based on a dynamic stability margin
}

\author{
G. Besseron, Ch. Grand, F. Ben Amar and Ph. Bidaud \\ Institut des Systèmes Intelligents et de Robotique (ISIR) \\ Université Pierre et Marie Curie - Paris VI, 75005 Paris, France \\ CNRS, FRE 2507, Paris, France \\ \{besseron, grand, amar, bidaud\}@isir.fr
}

\begin{abstract}
This paper concerns the control of an autonomous high mobility wheel-legged rover crossing uneven terrains. A new control strategy, using active redundancies of the robot, leads to elaborate a posture control based on the potential field approach of the stability measurement. Then a decoupled posture and trajectory control algorithm based on the velocity model of the robot is proposed. Last, simulation results showing performance of the control algorithm are presented.
\end{abstract}

\section{INTRODUCTION}

The main field of this research project deals with the mobility of autonomous robotic rovers moving on an unknown natural environment. Many potential applications like planetary or extreme environment (volcanic, arctic or desert) exploration, agriculture, defense, demining, and others various missions in hazardous areas can be considered. Therefore autonomous mobile robots must be able to move on a wide variety of terrains while ensuring the integrity of the system (i.e. the stability holding to avoid tipover). The main difficulties in this kind of environment are due to the geometrical and physical soil properties (large slopes, roughness, rocks distribution, soil compaction, friction characteristics, etc) - it is easier for an autonomous system to move on flat tar road than to cross a uneven stony field.

Lots of robotic systems have been developed in order to attempt to answer to these issues. Rovers such as Sojourner [1], Shrimp [2], or Nomad [3] are articulated multibody structures permitting a passive adaption to the ground surface. Other robotic systems like these of Gofor [4], SRR [5], Lama [6], Azimut [7], or MTR [8] use an active suspension allowing the control of some attitude parameters of the robot. High mobility hybrid systems - such as Hylos [9], Workpartner [10], or Athlete [11] robots - combine the advantages of both wheeled and legged vehicles - i.e. the ability to ensure some higher velocity than legged systems for the first one, and to cross terrains with high discontinuities (like rocks, steps, gaps, etc) for the second.

In this paper, we propose to analyze the problem of stability control for the Hylos robots which were developed in our lab (see Fig. 1) [9]. These rovers are high mobility redundantly actuated hybrid systems. They are lightweight (around twenty-five kilogrammes) robots with sixteen actively actuated degrees-of-freedom (four wheel/leg sub-assemblies, each one is made up of a two-degrees-of-freedom suspension
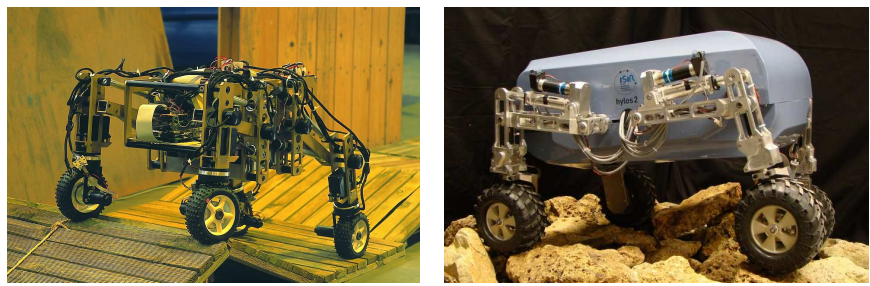

Fig. 1. Hylos I (left) and Hylos II (right)

mechanism and a steering and drive wheel). Because of active internal mobilities, the control of their posture could be considered. The problem of posture control for this kind of robotic system is a challenge regarding its complex dynamic interactions with the environment when moving on roughly irregular terrains.

Control approaches of such redundantly actuated systems have been proposed in previous works [4], [5]. They are usually based on the modeling and analysis of vehicle motion, and lead to improve the stability of the vehicle. In [9], authors propose a posture adjustment algorithm which controls the robot around a suboptimal posture, which, itself, optimizes both the traction force balance and the tipover margin for the Hylos robot. This suboptimal posture is defined when the Hylos robot is evolving on a sloping terrain, and corresponds to the case when the vertical components of the contact force are equally distributed.

In this study, the used strategy is different. Posture control consists in modifying the robot posture in order to ensure its stability without specifying strictly a postural state. The posture correction is so made only when the stability of wheel-legged vehicle is jeopardized. The proposed controller is based on the technique of "potential fields" for which an artificial potential, reflecting the rover tipover stability margin, is used.

In section II, after the introduction of the used stability margin, the potential field based on the stability measurement is proposed. Section III presents the formulation used to develop the differential kinematic model of a hybrid wheellegged robot. Next, decoupled posture and trajectory control algorithm is described in section IV. Finally, results of dynamic simulation to validate this new stability control strategy are shown in the last section $\mathrm{V}$. 


\section{Stability Margin and Linked Potential Field}

\section{A. Dynamic Stability Margin}

The control of robotic systems under stability margin conditions was mainly addressed in the field of legged locomotion. Since the first stability criterion [12] estimating stability for machine walking at constant speed on flat, even terrain, many of these stability criteria have been developed to adapt in more complex cases.

The control method presented in this paper considers the vehicle movement on an irregular terrain without discontinuities. Thus, the tipover stability margin is mainly constrained by the terrain geometry. In order to ensure the integrity of the vehicle during its navigation stage on an uneven terrain, its stability margin index must be estimated all the time. The "tipover stability margin" proposed by Papadopoulos and Rey [13] is used.

This tipover stability margin takes into account both the distance of the projected center-of-gravity (c.o.g) to the support polygon and its vertical position relatively to the average plane defined by contact points $C_{i}$. Moreover, all the external forces working on the c.o.g of the vehicle, including gravity, are considered. The formalism can be described briefly as follows (see Fig. 2): the line joining two consecutive terrain-contact points $C_{i}$ defines a tipover axis $\mathbf{a}_{i}$. The unit vector $\mathbf{h}_{i}$ of the axis, joining the vehicle c.o.g $G$ to the center of each tipover axis, is computed. Then, the angle $v_{i}$ between each $\mathbf{h}_{i}$ and the tipover contribution $\boldsymbol{f}_{i}^{*}$, computed from the total external force $\boldsymbol{\tau}_{\boldsymbol{t}}=\left\{\boldsymbol{f}_{\boldsymbol{t}}, \boldsymbol{m}_{\boldsymbol{t}}\right\}$ applied to the vehicle, gives the stability angle over the corresponding tipover axis. The system dynamics has been considered. A preliminary study has shown that the inertial terms of the central body of the robot prevail. The stability angle $v_{i}$ is therefore defined as the angle between $\mathbf{h}_{i}$ and the total external force $\tau_{t}$ including the gravitational and inertial forces. The overall vehicle stability margin $m_{s}$ is also defined as the minimum of all stability angles $v_{i}$ :

$$
m_{s}=\min \left(v_{i}\right) \text { for } i=\{1, \ldots, n\}
$$

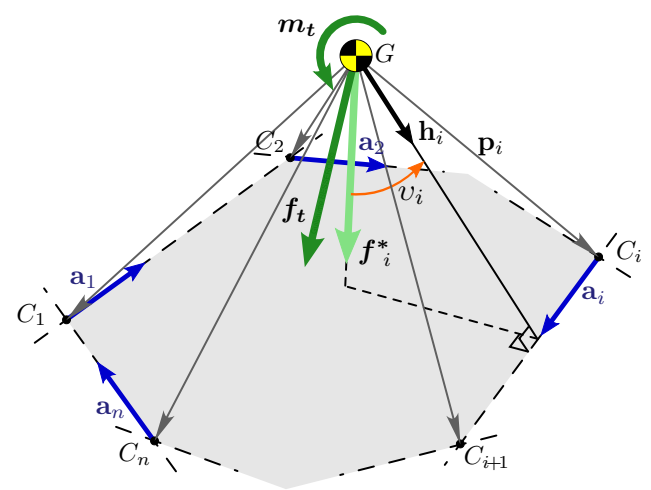

Fig. 2. Used Stability Margin

\section{B. The Artificial Potential Field Approach}

The idea of the artificial potential field approach is to consider that the robot moves in a field of forces. Each force,
$\boldsymbol{F}_{\boldsymbol{i}}$ is parallel to the opposite gradient of some potential field, $U_{i}$

$$
\boldsymbol{F}_{\boldsymbol{i}}=-\nabla U_{i}
$$

This method has been described by Khatib [14] for the first time in 1986 and has been often used since. This approach is very flexible and allows to consider many and varied kind of constraints. The total potential field $U$ can result from numerous functions $U_{i}$ defined by:

$$
U=\sum \alpha_{i} U_{i}
$$

where $U_{i}$ express the potential functions for obstacle avoidance, path tracking [15], joint limit avoidance or, in the present case, to guarantee the stability of the system.

The potential field could be either attractive $U_{i_{\text {[att] }}}$ or repulsive $U_{i_{\text {[rep] }}}$. Both forms are respectively depicted in figures 3(a) and 3(b):

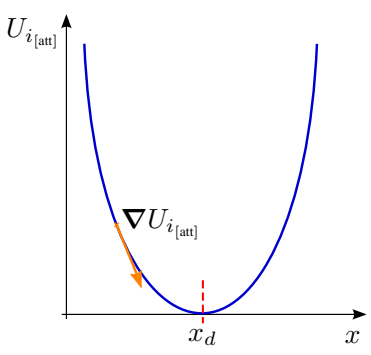

(a) Attractive

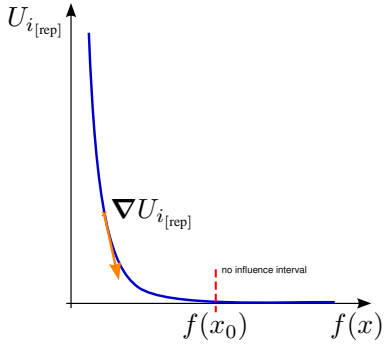

(b) Repulsive
Fig. 3. Both forms of potential functions

\section{Stability Potential Field}

To get a differential form for the potential field (as it is required), the stability potential function relative to the stability margin $U_{s t a b}$ is not directly issued from the stability margin $m_{s}$. In fact a potential function $U_{\text {stab }_{i}}$ is defined for each tipover axis corresponding to each stability angle $v_{i}$. The stability potential function $U_{s t a b}$ results from the sum of each $U_{s t a b_{i}}$, of which the specific form of repulsive potential function has been chosen in accordance with the potential field approach.

$$
U_{s t a b}(\mathbf{q})=\sum_{i} U_{\text {stab }_{i}}(\mathbf{q})
$$

with

$$
U_{s t a b_{i}}(\mathbf{q})=\left\{\begin{array}{cl}
\frac{1}{2} k_{\text {stab }}\left(\frac{1}{v_{i}(\mathbf{q})}-\frac{1}{v^{*}}\right)^{2} & \text { if } v_{i} \leq v^{*} \\
0 & \text { if } v_{i}>v^{*}
\end{array}\right.
$$

where $v_{i}$ is the stability angle or tipover angle relative to $i^{\text {th }}$ tipover axis, $v^{*}$ is the stability angle limit. Thus, the threshold of stability measurement from action must be defined in order to maintain an acceptable stability. $k_{s t a b}$ is a constant gain.

This function has a zero-band as shown on the figure 3(b), what results in having a correction in the robot control only when necessary. 


\section{Differential Kinematic Model}

In this paper, the same formalism as the one defined on previous works ([9], [16]) is used and adapted to the specific differential kinematics of the Hylos II robot.

For this class of system, a robot is composed of a main body connected to serial articulated chains ended by a cylindrical wheel. Let us define $\mathcal{R}_{0}$ the fixed frame, $\mathcal{R}_{P}$ the platform frame, $\mathcal{R}_{C_{i}}$ the contact frame for each wheel and $\mathcal{R}_{4_{i}}$ the frame attached to the $i^{\text {th }}$ rotating wheel (see Figs. 4 and 5).

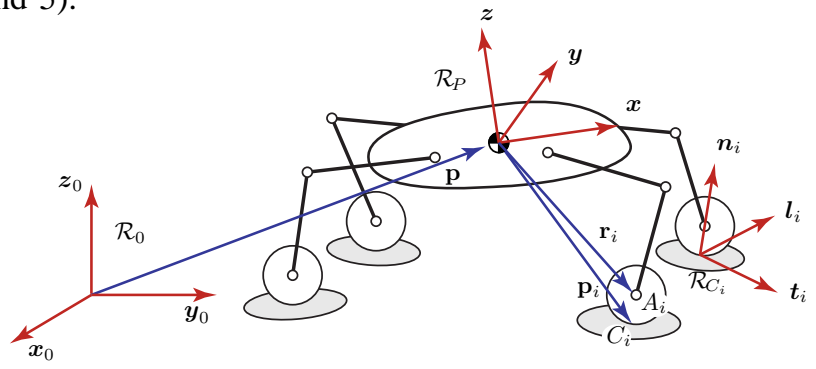

Fig. 4. General differential kinematical model of Hylos robot

The operational configuration of the robot is defined by $\mathbf{p}=(x, y, z)^{t}$ and $\phi=(\varphi, \psi, \theta)^{t}$ respectively the position and the orientation of the main body with respect to the fixed frame $\mathcal{R}_{0}$.

First, the velocity of the contact point due to the platform motion with respect to the ground and expressed in the platform frame $\mathcal{R}_{P}$ can be written as:

$$
\mathbf{v}_{\mathbf{x}}=\mathbf{R} \dot{\mathbf{p}}+\boldsymbol{\omega} \times \mathbf{p}_{i}
$$

where $\dot{\mathbf{p}}$ is the platform velocity expressed in $\mathcal{R}_{0}$ and $\boldsymbol{\omega}$ is the platform rotation velocity vector expressed in $\mathcal{R}_{P}$. $\mathbf{R}$ is the rotation matrix between the platform frame and the ground one and $\mathbf{p}_{i}$ is the position of the contact point in the platform frame.

The vector $\mathbf{p}_{i}$ depends on leg parameters $\mathbf{q}_{i}$ (including $q_{1_{i}}$ and $q_{2_{i}}$ ). It is obtained by writing the kinematic model of the leg:

$$
\mathbf{p}_{i}=\mathcal{G}_{i}\left(\mathbf{q}_{i}\right)
$$

Then equation (6) can be rewritten in a matrix form:

$$
\begin{aligned}
\mathbf{v}_{\mathbf{x}} & =\mathbf{R} \dot{\mathbf{p}}-\widetilde{\mathbf{p}}_{i} \mathbf{T}_{\phi} \dot{\phi} \\
& =\left[\mathbf{R}-\widetilde{\mathbf{p}}_{i} \mathbf{T}_{\phi}\right] \dot{\mathbf{x}} \\
& =\mathbf{L}_{i} \dot{\mathbf{x}}
\end{aligned}
$$

where $\widetilde{\mathbf{p}_{i}}$ is the skew matrix corresponding to the crossproduct, $\dot{\mathbf{x}}$ is the platform velocity twist with respect to the ground frame $\mathcal{R}_{0}$ and $\mathbf{T}_{\phi}$ is the rotation velocity decoupling matrix, detailed in appendix. This matrix expresses the rotation velocity vector $\boldsymbol{\omega}$ as a function of the rotation angle derivative $\dot{\phi}$. $\mathbf{L}_{i}$ is called locomotion matrix with a $3 \times 6$ dimensions.

The velocity of the contact point $C_{i}$ due to the leg motion with respect to the platform is expressed by a classical serial chain differential kinematic model:

$$
\begin{aligned}
\mathbf{v}_{\mathbf{p}_{i}} & =\mathbf{J}_{\mathbf{p}_{i}} \dot{\mathbf{q}}_{i} \\
& =\left[\begin{array}{lll}
\boldsymbol{\sigma}_{1} \times \mathbf{a}_{1} & \ldots & \boldsymbol{\sigma}_{m} \times \mathbf{a}_{m}
\end{array}\right] \dot{\mathbf{q}}_{i}
\end{aligned}
$$

The differential kinematic model is obtained by means of the velocity composition principle expressed in the contact frame $\mathcal{R}_{C_{i}}$ :

$$
\mathbf{v}_{\mathbf{s}}=-\mathbf{v}_{\mathbf{c}}+\mathbf{v}_{\mathbf{p}_{i}}+\mathbf{v}_{\mathbf{x}}
$$

where:

- $\mathbf{v}_{\mathrm{s}}$ is the sliding velocity of the contact point $C_{i}$,

- $\mathbf{v}_{\mathbf{x}}$ is the velocity of $C_{i}$ due to platform motion with respect to ground,

- $\mathbf{v}_{\mathbf{p}_{i}}$ is the velocity of $C_{i}$ due to leg's motion with respect the platform,

- $\mathbf{v}_{\mathbf{c}}=r \omega_{i} \boldsymbol{t}_{i}$ is the wheel circumferential velocity with respect to the leg.

On the assumption of pure rolling (slip velocity is null), we obtain then from equation (10) by projection on the contact frame $\mathcal{R}_{C_{i}}=\left(C_{i}, \boldsymbol{t}_{i}, \boldsymbol{l}_{i}, \boldsymbol{n}_{i}\right)$ :

$$
\mathbf{R}_{i}{ }^{t} \mathbf{L}_{i} \dot{\mathbf{x}}+\mathbf{R}_{i}{ }^{t} \mathbf{J}_{\mathbf{p}_{i}} \dot{\mathbf{q}}_{i}-r \omega_{i} \boldsymbol{t}_{i}=\mathbf{0}
$$

where $\mathbf{R}_{i}$ is the matrix rotation of the contact frame with respect to the platform frame and $\omega_{i}$ is the $i^{\text {th }}$ wheel rate.

Finally, we obtain, in a matrix-form, the velocity equation for the whole system composed of four wheel-legged chains:

$$
\mathbf{L} \dot{\mathbf{x}}+\mathbf{J} \dot{\mathbf{q}}=\mathbf{0}
$$

where $\mathbf{L}$ is the locomotion matrix which gives the wheel contribution to the platform movement, $\mathbf{J}$ corresponds to the Jacobian matrix of wheel-legged differential kinematic chain, and where $\mathbf{x}$ and $\mathbf{q}$ are respectively the vectors of the platform parameters and the articular-joint parameters of wheel-legged chains.

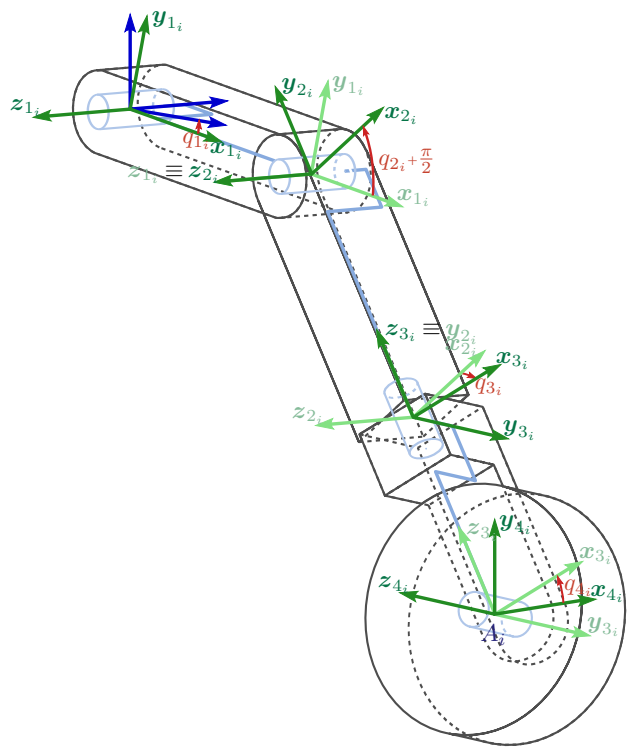

Fig. 5. One wheel-legged structure scheme of Hylos robot

\section{Decoupled Control}

The motion control of the studied redundant system is based on the resolution of the inverse velocity model. Several classical approaches of redundancies control and issued from manipulators control have been considered ([17], [18], [19], 


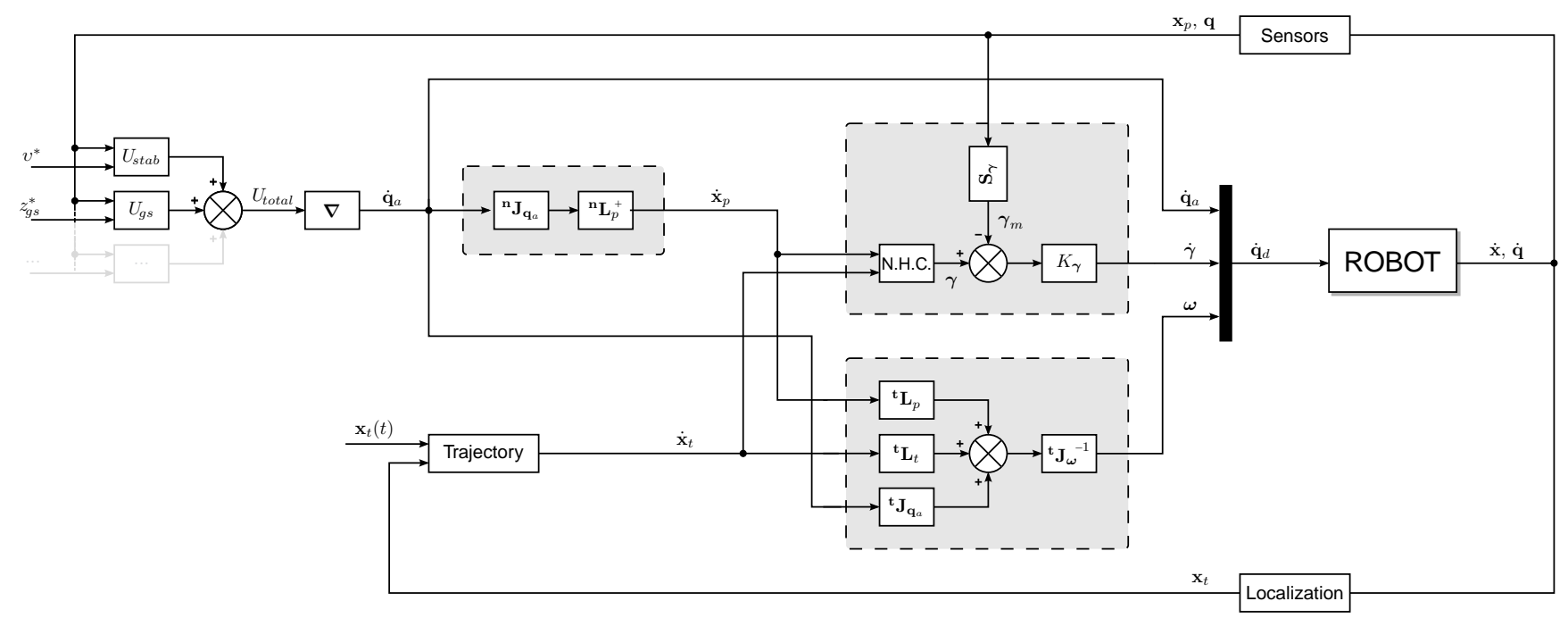

Fig. 6. Control scheme

[20]). In order to solve the inverse model of equation (12), the task in the operational space is defined following two modalities: one relative to the robot posture and the other to the trajectory control.

Thus the vector of platform velocities $\dot{\mathbf{x}}=(\dot{x}, \dot{y}, \dot{z}, \dot{\varphi}, \dot{\psi}, \dot{\theta})^{t}$ - input of inverse model - is split into two sub-vectors $\dot{\mathbf{x}}_{t}$ and $\dot{\mathbf{x}}_{p}$. Each of them put together respectively the trajectory and posture terms:

$$
\left\{\begin{array}{l}
\dot{\mathbf{x}}_{t}=(\dot{x}, \dot{y}, \dot{\theta})^{t}=\mathbf{S}_{t} \dot{\mathbf{x}} \\
\dot{\mathbf{x}}_{p}=(\dot{z}, \dot{\varphi}, \dot{\psi})^{t}=\mathbf{S}_{p} \dot{\mathbf{x}}
\end{array}\right.
$$

where $\mathbf{S}_{t}$ and $\mathbf{S}_{p}$ are the appropriate sorting matrices, which are detailed in appendix.

The trajectory following task is realized by controlling the velocity term $\dot{\mathbf{x}}_{t}$ using a classical control approach. Then, the problem is to compute the joint velocity term $\dot{\mathbf{q}}$ in order to obtain the desired $\dot{\mathbf{x}}_{t}$ while ensuring the robot stability using a decoupled inverse differential kinematic model.

Furthermore, the actuated joint velocities are also split into three groups considering their effects on the reference body velocity:

$$
\dot{\mathbf{q}}=\left(\dot{\mathbf{q}}_{a}, \dot{\gamma}, \boldsymbol{\omega}\right)^{t}
$$

where $\dot{\mathbf{q}}_{a}$ groups together the set of the leg joints, $\dot{\gamma}$ the wheel steering joint and $\boldsymbol{\omega}$ the wheel spin velocity, which are respectively introduced by the associated sorting matrix, explained in appendix:

$$
\dot{\mathbf{q}}_{a}=\mathbf{S}_{\mathbf{q}_{a}} \dot{\mathbf{q}} \quad \dot{\gamma}=\mathbf{S}_{\gamma} \dot{\mathbf{q}} \quad \boldsymbol{\omega}=\mathbf{S}_{\boldsymbol{\omega}} \dot{\mathbf{q}}
$$

Posture motion analysis: The projection of the differential kinematic constraints on each contact normal $\boldsymbol{n}_{i}$ leads to eliminate the $\dot{\gamma}$ and $\boldsymbol{\omega}$ velocities in the kinematic equation as they do not contribute to the robot motion in this direction:

$$
\mathbf{P}_{\mathbf{n}}\left[\mathbf{L}\left(\mathbf{S}_{t}^{t} \mathbf{S}_{t} \dot{\mathbf{x}}+\mathbf{S}_{p}^{t} \mathbf{S}_{p} \dot{\mathbf{x}}\right)+\mathbf{J}\left(\mathbf{S}_{\mathbf{q}_{a}}{ }^{t} \mathbf{S}_{\mathbf{q}_{a}} \dot{\mathbf{q}}\right)\right]=\mathbf{0}
$$

where $\mathbf{P}_{\mathbf{n}}$ is the projection matrix associated to $\mathbf{n}$, the set of the vectors $\boldsymbol{n}_{i} . \mathbf{P}_{\mathbf{n}}$ is also defined in appendix.

Then, considering that the leg motion marginally contributes to the reference body motion with respect to the wheel rotation, the term $\mathbf{P}_{\mathbf{n}} \mathbf{L}\left(\mathbf{S}_{t}{ }_{t} \mathbf{S}_{t} \dot{\mathbf{x}}\right)$ is negligible with respect to the term $\mathbf{P}_{\mathbf{n}} \mathbf{L}\left(\mathbf{S}_{p}^{t} \mathbf{S}_{p} \dot{\mathbf{x}}\right)$. Finally, the relation (16) comes down to:

$$
{ }^{\mathbf{n}} \mathbf{L}_{p} \dot{\mathbf{x}}_{p}+{ }^{\mathbf{n}} \mathbf{J}_{\mathbf{q}_{a}} \dot{\mathbf{q}}_{a}=\mathbf{0}
$$

$$
\text { with }{ }^{\mathbf{n}} \mathbf{L}_{p}=\left(\mathbf{P}_{\mathbf{n}} \mathbf{L} \mathbf{S}_{p}^{t}\right) \text { and }{ }^{\mathbf{n}} \mathbf{J}_{\mathbf{q}_{a}}=\left(\mathbf{P}_{\mathbf{n}} \mathbf{J} \mathbf{S}_{\mathbf{q}_{a}}{ }^{t}\right) \text {. }
$$

Trajectory motion analysis: The same analysis can be conducted by considering then the projection of the differential kinematic constraints on each longitudinal contact vector $\boldsymbol{t}_{i}$ and last on each lateral contact vector $\boldsymbol{l}_{i}$ :

$$
\begin{gathered}
{ }^{\mathrm{t}} \mathbf{L} \dot{\mathbf{x}}+{ }^{\mathrm{t}} \mathbf{J}_{\mathbf{q}_{a}} \dot{\mathbf{q}}_{a}+{ }^{\mathbf{t}} \mathbf{J}_{\boldsymbol{\omega}} \boldsymbol{\omega}=\mathbf{0} \\
{ }^{\mathbf{l}} \mathbf{L} \dot{\mathbf{x}}+{ }^{\mathbf{l}} \mathbf{J}_{\mathbf{q}_{a}} \dot{\mathbf{q}}_{a}=\mathbf{0}
\end{gathered}
$$

The term $\mathbf{J}_{\gamma} \dot{\gamma}$ is neglected in the differential kinematic equations by considering that the castor angle is very small during the robot motion. Thus, $\dot{\gamma}$ the steering velocity as no effect on the instantaneous reference body velocity $\dot{x}$. So, $\gamma$ is determined by the equation (19) that corresponds here to the non-holonomic constraints (N.H.C.).

Once this decoupled analysis is established, the solving method is set up. The desired leg joints $\dot{q}_{a}$ are substituted for the potential field gradient $\nabla U$, established previously in order to control the robot posture (see Sec. II):

$$
\begin{aligned}
\dot{\mathbf{x}}_{p} & =-\left({ }^{\mathbf{n}} \mathbf{L}_{p}\right)^{+}{ }^{\mathbf{n}} \mathbf{J}_{\mathbf{q}_{a}} \dot{\mathbf{q}}_{a} \\
& =-\left({ }^{\mathbf{n}} \mathbf{L}_{p}\right)^{+\mathbf{n}} \mathbf{J}_{\mathbf{q}_{a}} \nabla U
\end{aligned}
$$

where $\left({ }^{\mathbf{n}} \mathbf{L}_{p}\right)^{+}$represents the pseudo-inverse matrix of ${ }^{\mathbf{n}} \mathbf{L}_{p}$.

Lastly, the trajectory control leads to compute the wheel velocity $\boldsymbol{\omega}$ and the steering velocity $\dot{\gamma}$. The first is stemmed from the relation (18) where the posture control $\dot{\mathbf{x}}_{p}$, issued of the relation (20), and the desired trajectory $\dot{\mathbf{x}}_{t}$ are inputed into the relation (21). As previously, $\dot{q}_{a}$ is substituted for the potential field gradient $\nabla U$ :

$$
\boldsymbol{\omega}=-\left({ }^{\mathbf{t}} \mathbf{J}_{\boldsymbol{\omega}}\right)^{-1}\left({ }^{\mathbf{t}} \mathbf{L}_{t} \dot{\mathbf{x}}_{t}+{ }^{\mathbf{t}} \mathbf{L}_{p} \dot{\mathbf{x}}_{p}+{ }^{\mathbf{t}} \mathbf{J}_{\mathbf{q}_{a}} \nabla U\right)
$$

The second comes from the compatible steering angle $\gamma_{i}$, computed from the non-holonomic equation (19).

$$
\dot{\gamma}=\left\{\dot{\gamma}_{i}\right\} \quad \text { with } \quad \dot{\gamma}_{i}=K_{\gamma}\left(\gamma_{i}-\gamma_{i_{m}}\right)
$$

where $\gamma_{i_{m}}$ is the measure of the steering angle, and $K_{\gamma}$ is a gain control.

The whole posture control algorithm is summed up through the control scheme depicted in figure 6 . 


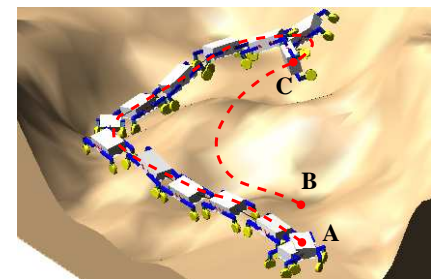

(a) without Control

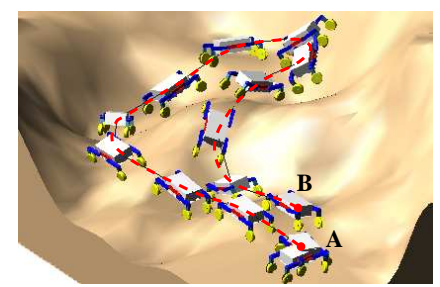

(b) with Control
Fig. 7. Hylos robot moving on an uneven terrain with a "loop" trajectory

\section{RESUlts}

The proposed posture control algorithm has been evaluated in dynamic simulation in order to validate its running principle. As shown in figure 7, this one has consisted in modeling the dynamic behaviour of the Hylos robot moving on an uneven ground in following a "loop" trajectory (from point "A" to point "B"). The results of this simulation are presented through figures 9 and 10, which depict respectively the evolution of the stability margin and the global potential generated for the posture correction. Figure 8 allows to observe the evolution of the robot joint angles during the whole simulation.

The first observation we have made is that the robot tips over when the posture control is not activated (see Fig. 7(a)). This stability loss is depicted on the figure 9 when the stability margin becomes negative. The point " $\mathrm{C}$ " on figures 7(a) and 9 shows this moment.

On the contrary, when the posture control is engaged the robot completes the set trajectory in spite of the elevation terrain variations. The analysis of the figures 8,9 and 10 shows there is a synchronisation between those variables. As planned by the control strategy, a posture correction of the robot is made when the stability margin drops under the stability margin limit $\left(v^{*}=0,35 \mathrm{rad}\right)$. Every time that this case appears, the potential function relative to the stability measurement becomes non-null (see Fig. 10). Then the robot posture is modified only if a potential field is generated (see Fig. 8). Thus the robot stability is preserved without imposing a specific posture.

\section{CONCLUSION}

In this paper, a new stability control strategy for a wheellegged robot has been proposed. This one has come from the idea to use active redundancies of the studied rover in order to ensure its stability without imposing a particular posture, as made in previous works [9]. This strategy needed to set up a decoupled control of posture and trajectory. An original velocity based control algorithm has been presented. This approach allows to carry out the desired behaviour of the robot. The algorithm has been validated through dynamic simulations, showing the capabilities of such a redundantly actuated robot to ensure both its stability margin during the whole motion on uneven terrain and a specified trajectory.

Experiments with the Hylos robot are in progress. Shortly the practical feasibility of this control approach will be evaluated and validated through these experiments.
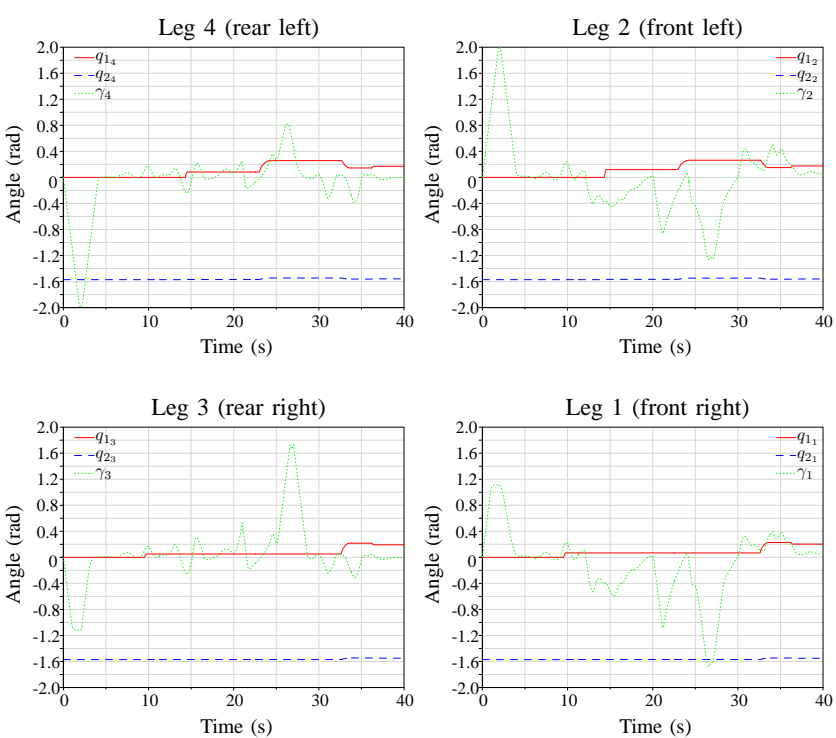

Fig. 8. Evolution of the joint angles $\left(q_{1_{i}}, q_{2_{i}}\right.$ and $\left.\gamma_{i}\right)$

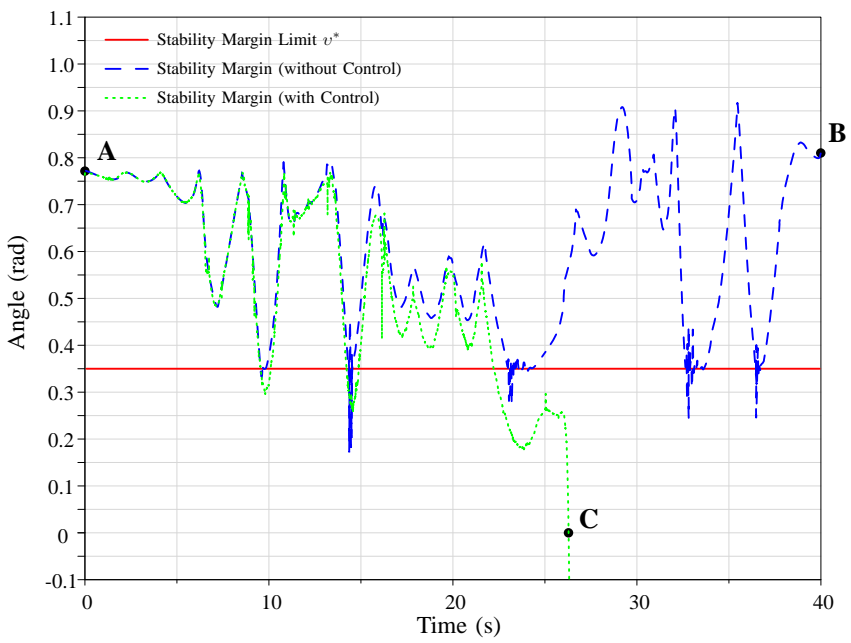

Fig. 9. Evolution of the stability margin

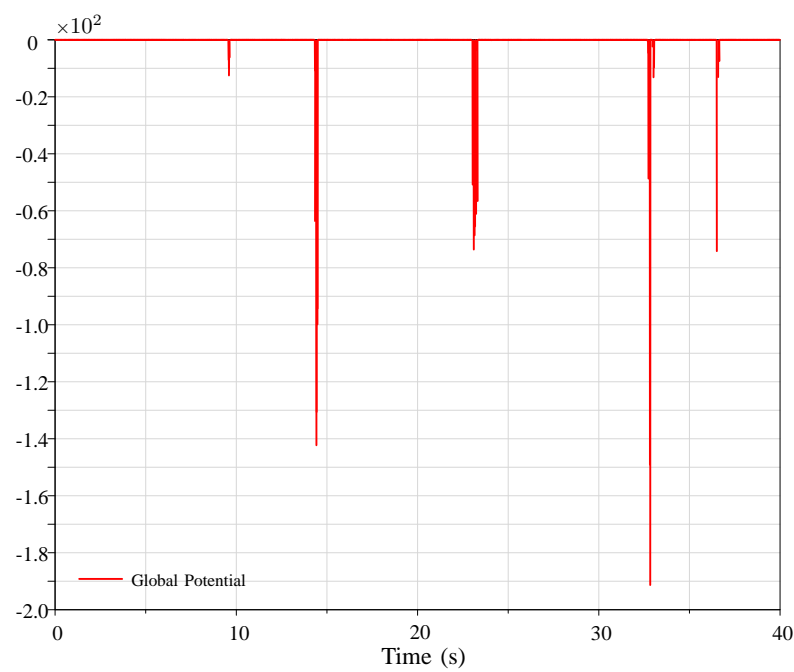

Fig. 10. Evolution of the global potential 


\section{APPENDIX}

\section{A. Differential Kinematics Matrices details}

$$
\boldsymbol{\omega}=\mathbf{T}_{\boldsymbol{\phi}} \dot{\boldsymbol{\phi}} \quad \text { with } \quad \mathbf{T}_{\boldsymbol{\phi}}(\boldsymbol{\phi})=\left(\begin{array}{ccc}
c_{\theta} c_{\psi} & -s_{\theta} & 0 \\
s_{\theta} c_{\psi} & c_{\theta} & 0 \\
-s_{\psi} & 0 & 1
\end{array}\right)_{[3 \times 3]}
$$

$$
\mathbf{J}=\left(\begin{array}{cccc}
\mathbf{J}_{1} & \mathbf{0} & \mathbf{0} & \mathbf{0} \\
\mathbf{0} & \mathbf{J}_{2} & \mathbf{0} & \mathbf{0} \\
\mathbf{0} & \mathbf{0} & \mathbf{J}_{3} & \mathbf{0} \\
\mathbf{0} & \mathbf{0} & \mathbf{0} & \mathbf{J}_{4}
\end{array}\right)_{[12 \times 16]}
$$

with

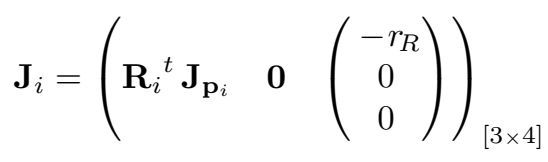

$$
\mathbf{L}=\left(\begin{array}{l}
\mathbf{R}_{1}^{t} \mathbf{L}_{1} \\
\mathbf{R}_{2}^{t} \mathbf{L}_{2} \\
\mathbf{R}_{3}^{t} \mathbf{L}_{3} \\
\mathbf{R}_{4}^{t} \mathbf{L}_{4}
\end{array}\right)_{[12 \times 6]}
$$

$$
\dot{\mathbf{x}}=(\dot{x}, \dot{y}, \dot{z}, \dot{\varphi}, \dot{\psi}, \dot{\theta})_{[6 \times 1]}^{t}
$$

$\dot{\mathbf{q}}=\left(\dot{q}_{1_{1}}, \dot{q}_{2_{1}}, \dot{\gamma}_{1}, \omega_{1}, \dot{q}_{1_{2}}, \dot{q}_{2_{2}}, \dot{\gamma}_{2}, \omega_{2}, \dot{q}_{1_{3}}, \dot{q}_{2_{3}}, \dot{\gamma}_{3}, \omega_{3}, \dot{q}_{1_{4}}, \dot{q}_{2_{4}}, \dot{\gamma}_{4}, \omega_{4}\right)_{[16 \times 1]}^{t}$

\section{B. Sorting Matrices details}

$$
\begin{aligned}
\mathbf{S}_{t} & =\left(\begin{array}{cccccc}
1 & 0 & 0 & 0 & 0 & 0 \\
0 & 1 & 0 & 0 & 0 & 0 \\
0 & 0 & 0 & 0 & 0 & 1
\end{array}\right)_{[3 \times 6]} \mathbf{S}_{p}=\left(\begin{array}{llllll}
0 & 0 & 1 & 0 & 0 & 0 \\
0 & 0 & 0 & 1 & 0 & 0 \\
0 & 0 & 0 & 0 & 1 & 0
\end{array}\right)_{[3 \times 6]} \\
\mathbf{S}_{\mathbf{q}_{a}} & =\left(\begin{array}{cccc}
\mathbf{S}_{\mathbf{q}_{1}} & \mathbf{0} & \mathbf{0} & \mathbf{0} \\
\mathbf{0} & \mathbf{S}_{\mathbf{q}_{2}} & \mathbf{0} & \mathbf{0} \\
\mathbf{0} & \mathbf{0} & \mathbf{S}_{\mathbf{q}_{3}} & \mathbf{0} \\
\mathbf{0} & \mathbf{0} & \mathbf{0} & \mathbf{S}_{\mathbf{q}_{4}}
\end{array}\right)_{[8 \times 16]} \text { with } \mathbf{S}_{\mathbf{q}_{i}}=\left(\begin{array}{llll}
1 & 0 & 0 & 0 \\
0 & 1 & 0 & 0
\end{array}\right) \\
\mathbf{S}_{\boldsymbol{\gamma}} & =\left(\begin{array}{cccc}
\mathbf{S}_{\gamma_{1}} \mathbf{0} & \mathbf{0} & \mathbf{0} \\
\mathbf{0} & \mathbf{S}_{\gamma_{2}} & \mathbf{0} & \mathbf{0} \\
\mathbf{0} & \mathbf{0} & \mathbf{S}_{\gamma_{3}} \mathbf{0} \\
\mathbf{0} & \mathbf{0} & \mathbf{0} & \mathbf{S}_{\gamma_{4}}
\end{array}\right)_{[4 \times 16]} \text { with } \mathbf{S}_{\gamma_{i}}=\left(\begin{array}{llll}
0 & 0 & 1 & 0
\end{array}\right) \\
\mathbf{S}_{\boldsymbol{\omega}} & =\left(\begin{array}{cccc}
\mathbf{S}_{\omega_{1}} & \mathbf{0} & \mathbf{0} & \mathbf{0} \\
\mathbf{0} & \mathbf{S}_{\omega_{2}} \mathbf{0} & \mathbf{0} \\
\mathbf{0} & \mathbf{0} & \mathbf{S}_{\omega_{3}} \mathbf{0} \\
\mathbf{0} & \mathbf{0} & \mathbf{0} & \mathbf{S}_{\omega_{4}}
\end{array}\right)_{[4 \times 16]} \text { with } \mathbf{S}_{\omega_{i}}=\left(\begin{array}{llll}
0 & 0 & 0 & 1
\end{array}\right)
\end{aligned}
$$

\section{Projection Matrices details}

$$
\begin{aligned}
& \mathbf{P}_{\mathbf{t}}=\left(\begin{array}{cccc}
\mathbf{P}_{\boldsymbol{t}_{1}} & \mathbf{0} & \mathbf{0} & \mathbf{0} \\
\mathbf{0} & \mathbf{P}_{\boldsymbol{t}_{2}} & \mathbf{0} & \mathbf{0} \\
\mathbf{0} & \mathbf{0} & \mathbf{P}_{\boldsymbol{t}_{3}} & \mathbf{0} \\
\mathbf{0} & \mathbf{0} & \mathbf{0} & \mathbf{P}_{\boldsymbol{t}_{4}}
\end{array}\right) \text { with } \quad \mathbf{P}_{\boldsymbol{t}_{i}}=\left(\begin{array}{lll}
1 & 0 & 0
\end{array}\right) \\
& \mathbf{P}_{\mathbf{l}}=\left(\begin{array}{cccc}
\mathbf{P}_{\boldsymbol{l}_{1}} & \mathbf{0} & \mathbf{0} & \mathbf{0} \\
\mathbf{0} & \mathbf{P}_{\boldsymbol{l}_{2}} & \mathbf{0} & \mathbf{0} \\
\mathbf{0} & \mathbf{0} & \mathbf{P}_{\boldsymbol{l}_{3}} & \mathbf{0} \\
\mathbf{0} & \mathbf{0} & \mathbf{0} & \mathbf{P}_{\boldsymbol{l}_{4}}
\end{array}\right)_{[4 \times 12]} \quad \text { with } \quad \mathbf{P}_{\boldsymbol{l}_{i}}=\left(\begin{array}{lll}
0 & 1 & 0
\end{array}\right) \\
& \mathbf{P}_{\mathbf{n}}=\left(\begin{array}{cccc}
\mathbf{P}_{\boldsymbol{n}_{1}} & \mathbf{0} & \mathbf{0} & \mathbf{0} \\
\mathbf{0} & \mathbf{P}_{\boldsymbol{n}_{2}} & \mathbf{0} & \mathbf{0} \\
\mathbf{0} & \mathbf{0} & \mathbf{P}_{\boldsymbol{n}_{3}} & \mathbf{0} \\
\mathbf{0} & \mathbf{0} & \mathbf{0} & \mathbf{P}_{\boldsymbol{n}_{4}}
\end{array}\right) \text { with } \quad \mathbf{P}_{\boldsymbol{n}_{i}}=\left(\begin{array}{lll}
0 & 0 & 1
\end{array}\right)
\end{aligned}
$$

\section{REFERENCES}

[1] R. Volpe, "Rocky 7: A next generation mars rover prototype," Journal of Advanced Robotics, vol. 11, no. 4, pp. 341-358, 1997.

[2] R. Siegwart, P. Lamon, T. Estier, M. Lauria, and R. Piguet, "Innovative design for wheeled locomotion in rough terrain," Robotics and Autonomous Systems, vol. 40, pp. 151-162, 2002.

[3] E. Rollins, J. Luntz, A. Foessel, B. Shamah, and W. Whittaker, "Nomad: A demonstration of the transforming chassis," in IEEE International Conference on Robotics and Automation, vol. 1, Leuven, Belgium, May 1998, pp. 611-617.

[4] S. Sreenivasan and B. Wilcox, "Stability and traction control of an actively actued micro-rover," Journal of Robotics Systems, vol. 11, no. 6, pp. 487-502, 1994.

[5] K. Iagnemma, A. Rzepniewski, S. Dubowsky, and P. Schenker, "Control of robotic vehicles with actively articulated suspensions in rough terrain," Autonomous Robots, vol. 14, no. 1, pp. 5-16, 2003.

[6] G. Andrade, F. BenAmar, P. Bidaud, and R. Chatila, "Modeling robotsoil interaction for planetary rover motion control," in IEEE/RS International Conference on Intelligent Robots and Systems, vol. 1, Victoria, B.C., Canada, October 1998, pp. 576-581.

[7] F. Michaud, D. Ltourneau, J.-F. Par, M.-A. Legault, R. Cadrin, M. Arsenault, Y. Bergeron, M.-C. Tremblay, F. Gagnon, M. Millette, P. Lepage, Y. Morin, and S. Caron, "Multi-modal locomotion robotic platform using leg-track-wheel articulations," Autonomous Robots, Special Issue on Unconventional Robotic Mobility, vol. 18, no. 2, pp. 137-156, March 2005.

[8] A. K. Bouloubasis and G. T. McKee, "The mobility system of the multi-tasking rover (mtr)," in International Conference on Robotics and Automation, Roma, Italy, April 2007, pp. 4919-4924.

[9] C. Grand, F. BenAmar, F. Plumet, and P. Bidaud, "Stability and traction optimization of a reconfigurable wheel-legged robot," The International Journal of Robotics Research, vol. 23, no. 10-11, pp. 1041-1058, October-November 2004.

[10] A. Halme, I. Leppänen, S. Salmi, and S. Ylönen, "Hybrid locomotion of a wheel-legged machine," in 3rd Int. Conference on Climbing and Walking Robots, 2000.

[11] B. H. Wilcox, T. Litwin, J. Biesiadecki, J. Matthews, M. Heverly, J. Morrison, J. Townsend, N. Ahmed, A. Sirota, and B. Cooper, "Athlete: A cargo handling and manipulation robot for the moon," Journal of Field Robotics, vol. 24, no. 5, pp. 421-434, April 2007.

[12] R. McGhee and A. Frank, "On the stability properties of quadruped creeping gait," Mathematical Bioscience, vol. 3, pp. 331-351, 1968.

[13] E. Papadopoulos and D. Rey, "A new mesure of tipover stability for mobile manipulators," in IEEE Int. Conf. on Robotics and Automation, ICRA'96, 1996, pp. 3111-3116.

[14] O. Khatib, "Real-time obstacle avoidance for manipulators and mobile robots," The International Journal of Robotics Research, vol. 5, no. 1, pp. 90-98, Spring 1986.

[15] M. Sørensen, "Artificial potential field approach to path tracking for a non-holonomic mobile robot," in 11th Mediterranean Conference on Control and Automation, Rhodes, Greece, 2003.

[16] C. Grand, F. B. Amar, and F. Plumet, "Motion kinematics analysis of wheeled-legged rover over 3d surface with posture adaptation," in 12th IFToMM World Congress in Mechanism and Machine Science, Besançon, France, June 2007.

[17] J. Baillieul, "Kinematic programming alternatives for redundant manipulators," in IEEE International Conference on Robotics and $\mathrm{Au}$ tomation, vol. 2, St Louis, March 1985, pp. 722-728.

[18] D. N. Nenchev, "Restricted jacobian matrices of redundant manipulators in constrained motion tasks," International Journal of Robotics Research, vol. 11, no. 6, pp. 584-597, 1992.

[19] C. A. Klein, C. Chu-Jenq, and S. Ahmed, "A new formulation of the extended jacobian method and its use in mapping algorithmic singularities for kinematically redundant manipulators," IEEE Transactions on Robotics and Automation, vol. 11, no. 1, pp. 50-55, 1995.

[20] J. Lenarčič, "Effective secondary task execution of redundant manipulators," in Robotica, C. U. Press, Ed., vol. 16, July 1998, pp. 457-462. 